\title{
Enaminone Complexes: Synthesis, Characterization and Bioactivity
}

\author{
BAKIR JERAGH and ABDEL-ZAHER A. ELASSAR* \\ Chemistry Department, Faculty of Science, Kuwait University, \\ P.O.Box 5969-Safat-13060, Kuwait \\ aelassar@yahoo.com
}

Received 11 October 2014 / Accepted 17 October 2014

\begin{abstract}
Synthesis of enaminones, viz., 3-(dimethylamino)-1-(thiophen-2-yl)prop-2-en-1-one and 3-(dimethylamino)-1-phenylprop-2-en-1-one, and their chelating with different metal nitrates of $\mathrm{Cu}^{2+}, \mathrm{Ni}^{2+}, \mathrm{Hg}^{2+}, \mathrm{Pb}^{2+}$ and $\mathrm{Co}^{2+}$ in ethnolic solution were reported. The bioactivity of ligands and metal complexes against eight types of bacteria and three types of fungi were investigated.
\end{abstract}

Keywords: Enaminone, Metal complex, Bioactivity

\section{Introduction}

Preparation and chemistry of enaminones have been reviewed by Elassar et al. ${ }^{1}$ Enaminones have received more attention during last decade due to its using as intermediate for many useful synthetic compounds in addition, a lot of work has been done to explore new routes for the synthesis and use of enaminones ${ }^{2-8}$. The presences of carbonyl group connected by carboncarbon double bond and an amino group or $N$-substituted amines in enaminones allow being act as bidentates system. The chemistry of carbonyl group in enaminone is an area of considerable scope ${ }^{9}$. Complexes of Fe(II) and Zn(II) ions with diethylamine-pent-3-ene-2-one, dipropylaminepent-3-ene-2-one and dicyclohexylamine-pent-3-ene-2-one are reported ${ }^{10} . \mathrm{BF}_{2}$ complex have been synthesized via reactions of 3-methylthioenaminones with $\mathrm{BF}_{3} \cdot \mathrm{Et}_{2} \mathrm{O}$ in the presence of $\mathrm{Et}_{3} \mathrm{~N}$ in good to excellent yields. ${ }^{11}$ In this article we are aimed to synthesis enaminones and use as a ligand for complexation with different metal ions. The biological activity of ligands and metal complexes are also aimed to be studied against gram positive, gram negative and fungi.

\section{Experimental}

Melting points were recorded on a Gallenkamp apparatus. IR spectra were recorded using $\mathrm{KBr}$ pellets on a JASCO FTIR-6300 FT-IR spectrophotometer. ${ }^{1} \mathrm{H}$ and ${ }^{13} \mathrm{C}$ NMR spectra were recorded on Bruker DPX AvanceII $600 \mathrm{MHz}$ super conducting NMR spectrometer with proton spectra measured at $600 \mathrm{MHz}$ and carbon spectra at $150 \mathrm{MHz}$, respectively. Mass spectra were measured on a high resolution GC/MS DFS-Thermo. Microanalyses were performed on Elementar-Vario Micro cube Analyzer. 


\section{Materials}

The starting materials and solvents used for the synthesis of ligands and complexes were used as received. The salts used are: $\mathrm{Co}\left(\mathrm{NO}_{3}\right)_{2} \cdot 6 \mathrm{H}_{2} \mathrm{O} ; \mathrm{Ni}\left(\mathrm{NO}_{3}\right)_{2} \cdot 6 \mathrm{H}_{2} \mathrm{O} ; \mathrm{Cu}\left(\mathrm{NO}_{3}\right)_{2} \cdot 3 \mathrm{H}_{2} \mathrm{O}$; $\mathrm{Cd}\left(\mathrm{NO}_{3}\right)_{2} \cdot 4 \mathrm{H}_{2} \mathrm{O} ; \mathrm{Hg}\left(\mathrm{NO}_{3}\right)_{2} \cdot \mathrm{H}_{2} \mathrm{O}$ and $\mathrm{Pb}\left(\mathrm{NO}_{3}\right)_{2}$.

\section{Synthesis of Ligand}

3-(Dimethylamino)-1-(thiophen-2-yl)prop-2-en-1-one (EN1); and 3-(dimethylamino) -1-phenylprop-2-en-1-one (EN2)

To a solution of 2-acetylthiophene or acetophenone $(0.01 \mathrm{~mol})$ in xylene $(30 \mathrm{~mL})$ dimethylformamide dimethylacetal, DMFDMA (0.01 mol) was added. The reaction mixture was heated under reflux for $8 \mathrm{~h}$. The solvent was evaporated under vacuum. After that the reaction product was treated with ice-cold water. The solid product obtained was collected by filtration and crystallized from the ethanol. (For elemental analysis and spectral data see Tables 1-3).

\section{Preparation of the complexes}

A solution of 3-(dimethylamino)-1-(thiophen-2-yl)prop-2-en-1-one or 3-(dimethylamino)-1phenylprop-2-en-1-one $(0.01 \mathrm{~mol})$ in $20 \mathrm{~mL}$ ethanol was added slowly with continuous stirring to a $10 \mathrm{~mL}$ of filtered aqueous ethanolic solution of the metal nitrate salt $(0.01 \mathrm{~mol})$ and then heated under reflux for $5 \mathrm{~h}$. The product was collected by filtration and allowed to evaporate slowly at room temperature. (For elemental analysis and spectral data see Tables 1-3).

Table 1. IR of EN1, EN2 and their complexes

\begin{tabular}{ll}
\hline Compound & IR $\left(\mathrm{cm}^{-1}\right)$ \\
\hline EN1 & $3072(\mathrm{CH}), 1653(\mathrm{CO}), 1545(\mathrm{C}-\mathrm{N})$ \\
$\mathrm{CuEN1}\left(\mathrm{NO}_{3}\right)_{2}$ & $3076(\mathrm{CH}), 1650(\mathrm{CO}), 1575(\mathrm{C}-\mathrm{N})$ \\
$\mathrm{Co}(\mathrm{EN} 1)_{4}\left(\mathrm{NO}_{3}\right)_{2}$ & $3097(\mathrm{CH}), 1654(\mathrm{CO}), 1588(\mathrm{C}-\mathrm{N})$ \\
$\mathrm{Ni}(\mathrm{EN} 1)_{3}\left(\mathrm{NO}_{3}\right)_{2} \cdot 2 \mathrm{H}_{2} \mathrm{O}$ & $3445\left(\mathrm{H}_{2} \mathrm{O}\right), 3096(\mathrm{CH}), 1652(\mathrm{CO}), 1587(\mathrm{C}-\mathrm{N})$ \\
$\mathrm{CdEN} 1\left(\mathrm{NO}_{3}\right)_{2}$ & $3551,3448\left(\mathrm{H}_{2} \mathrm{O}\right), 1657(\mathrm{CO}), 1594(\mathrm{C}-\mathrm{N})$ \\
$\mathrm{HgEN} 1\left(\mathrm{NO}_{3}\right)_{2}$ & $3428\left(\mathrm{H}_{2} \mathrm{O}\right), 1654(\mathrm{CO}), 1563(\mathrm{C}-\mathrm{N})$ \\
PbEN1 $\left(\mathrm{NO}_{3}\right)_{2}$ & $3093(\mathrm{CH}), 1653(\mathrm{CO}), 16191588(\mathrm{C}-\mathrm{N})$ \\
EN2 & $3054,3020(\mathrm{CH}), 1650(\mathrm{CO}), 1583(\mathrm{C}-\mathrm{N})$ \\
$\mathrm{CuEN2}\left(\mathrm{NO}_{3}\right)_{2}$ & $3062,3011(\mathrm{CH}), 1653(\mathrm{CO}), 1567(\mathrm{C}-\mathrm{N})$ \\
$\mathrm{Co}(\mathrm{EN2})_{4}\left(\mathrm{NO}_{3}\right)_{2}$ & $3062(\mathrm{CH}), 1655(\mathrm{CO}), 1570(\mathrm{C}-\mathrm{N})$ \\
$\mathrm{Ni}(\mathrm{EN} 2)_{4}\left(\mathrm{NO}_{3}\right)_{2} \cdot 3 \mathrm{H}_{2} \mathrm{O}$ & $3443\left(\mathrm{H}_{2} \mathrm{O}\right), 3063(\mathrm{CH}), 1651(\mathrm{CO}), 1571(\mathrm{C}-\mathrm{N})$ \\
$\mathrm{Cd}(\mathrm{EN} 2)\left(\mathrm{NO}_{3}\right)_{2} \cdot 2 \mathrm{H}_{2} \mathrm{O}$ & $3445(\mathrm{H} 2 \mathrm{O}), 3081(\mathrm{CH}), 1652(\mathrm{CO}), 1575(\mathrm{C}-\mathrm{N}$ \\
$\mathrm{Hg}(\mathrm{EN2})_{2}\left(\mathrm{NO}_{3}\right)_{2} \cdot 10 \mathrm{H}_{2} \mathrm{O}$ & $3445(\mathrm{H} 2 \mathrm{O}), 3053(\mathrm{CH}), 1657(\mathrm{CO}), 1565(\mathrm{C}-\mathrm{N})$ \\
$\mathrm{Pb}(\mathrm{EN} 1)_{2}\left(\mathrm{NO}_{3}\right)_{2}$ & $3050(\mathrm{CH}), 1659(\mathrm{CO}), 1596(\mathrm{C}-\mathrm{N})$ \\
\hline
\end{tabular}

Table 2. Formula, MS, melting point and elemental analysis of EN1, EN2 and their complexes

\begin{tabular}{llllllll}
\hline \multirow{2}{*}{ Compound } & \multicolumn{1}{c}{$\begin{array}{c}\text { Formula } \\
(\mathrm{M} . \mathrm{Wt})\end{array}$} & $\begin{array}{c}\mathrm{MS} \\
\mathrm{m} / \mathrm{z}\end{array}$ & $\begin{array}{c}\mathrm{Mp} \\
{ }^{\circ} \mathrm{C}\end{array}$ & $\begin{array}{c}\mathrm{C} \% \\
\mathrm{Calcd} / \\
\text { found }\end{array}$ & $\begin{array}{c}\mathrm{H} \% \\
\mathrm{Calcd} / \\
\text { found }\end{array}$ & $\begin{array}{c}\mathrm{N} \% \\
\text { Calcd/ } \\
\text { found }\end{array}$ & $\begin{array}{c}\mathrm{S} \% \\
\text { Calcd/ } \\
\text { found }\end{array}$ \\
\hline \multirow{2}{*}{$\mathrm{EN} 1$} & $\mathrm{C}_{9} \mathrm{H}_{11} \mathrm{NOS}$ & \multirow{2}{*}{181} & \multirow{2}{*}{110} & 59.64 & 6.12 & 7.73 & 17.69 \\
& $(181.25)$ & & & 59.74 & 5.99 & 8.06 & 17.41 \\
$\mathrm{CuEN1}\left(\mathrm{NO}_{3}\right)_{2}$ & $\mathrm{C}_{9} \mathrm{H}_{11} \mathrm{CuN}_{3} \mathrm{O}_{7} \mathrm{~S}$ & \multirow{2}{*}{369} & \multirow{2}{*}{135} & 29.31 & 3.01 & 11.39 & 8.69 \\
& $(368.81)$ & & & 29.44 & 3.03 & 11.42 & 8.83 \\
\hline
\end{tabular}




\begin{tabular}{|c|c|c|c|c|c|c|c|}
\hline \multirow{2}{*}{$\mathrm{Co}(\mathrm{EN} 1)_{4}\left(\mathrm{NO}_{3}\right)_{2}$} & $\mathrm{C}_{36} \mathrm{H}_{44} \mathrm{CoN}_{6} \mathrm{O}_{10}$ & \multirow{2}{*}{908} & \multirow{2}{*}{175} & 47.62 & 4.88 & 9.26 & 14.13 \\
\hline & $\mathrm{S}_{4}(907.96)$ & & & 47.99 & 4.70 & 9.56 & 14.48 \\
\hline \multirow{2}{*}{$\mathrm{Ni}(\mathrm{EN} 1)_{3}\left(\mathrm{NO}_{3}\right)_{2} \cdot 2 \mathrm{H}_{2} \mathrm{O}$} & $\mathrm{C}_{27} \mathrm{H}_{37} \mathrm{~N}_{5} \mathrm{NiO}_{11} \mathrm{~S}$ & \multirow{2}{*}{762} & \multirow{2}{*}{150} & 42.53 & 4.89 & 9.18 & 12.62 \\
\hline & $3(762.50)$ & & & 42.23 & 4.78 & 9.45 & 12.60 \\
\hline \multirow{2}{*}{$\mathrm{CdEN} 1\left(\mathrm{NO}_{3}\right)_{2}$} & $\mathrm{C}_{9} \mathrm{H}_{11} \mathrm{~N}_{3} \mathrm{NiO}_{7} \mathrm{~S}$ & \multirow{2}{*}{418} & \multirow{2}{*}{$>250$} & 25.88 & 2.65 & 10.06 & 7.68 \\
\hline & $(417.68)$ & & & 25.78 & 3.01 & 9.98 & 7.46 \\
\hline \multirow{2}{*}{$\operatorname{HgEN} 1\left(\mathrm{NO}_{3}\right)_{2}$} & $\mathrm{C}_{9} \mathrm{H}_{11} \mathrm{HgN}_{3} \mathrm{O}_{7} \mathrm{~S}$ & \multirow{2}{*}{506} & \multirow{2}{*}{$>250$} & 21.37 & 2.19 & 8.31 & 6.34 \\
\hline & $(505.85)$ & & & 20.95 & 2.26 & 8.58 & 6.05 \\
\hline \multirow{2}{*}{$\mathrm{PbEN} 1\left(\mathrm{NO}_{3}\right)_{2}$} & $\mathrm{C}_{9} \mathrm{H}_{11} \mathrm{~N}_{3} \mathrm{O}_{7} \mathrm{PbS}$ & \multirow{2}{*}{513} & \multirow{2}{*}{190} & 21.09 & 2.16 & 8.20 & 6.26 \\
\hline & $(512.46)$ & & & 21.36 & 2.35 & 8.47 & 6.18 \\
\hline \multirow{2}{*}{ EN2 } & $\mathrm{C}_{11} \mathrm{H}_{13} \mathrm{NO}$ & \multirow{2}{*}{175} & \multirow{2}{*}{85} & 75.40 & 7.48 & 7.99 & \\
\hline & 175.23 & & & 75.12 & 7.45 & 8.13 & \\
\hline \multirow{2}{*}{$\mathrm{CuEN} 2\left(\mathrm{NO}_{3}\right)_{2}$} & $\mathrm{C}_{11} \mathrm{H}_{13} \mathrm{CuN}_{3} \mathrm{O}_{7}$ & \multirow{2}{*}{363} & \multirow{2}{*}{165} & 36.42 & 3.61 & 11.58 & \\
\hline & 362.78 & & & 36.24 & 3.56 & 11.85 & \\
\hline \multirow{2}{*}{$\mathrm{Co}(\mathrm{EN} 2)_{4}\left(\mathrm{NO}_{3}\right)_{2}$} & $\mathrm{C}_{44} \mathrm{H}_{52} \mathrm{CoN}_{6} \mathrm{O}_{10}$ & \multirow{2}{*}{884} & \multirow{2}{*}{$>250$} & 59.79 & 5.93 & 9.51 & \\
\hline & 883.85 & & & 59.65 & 5.65 & 9.31 & \\
\hline \multirow{2}{*}{$\mathrm{Ni}(\mathrm{EN} 2)_{4}\left(\mathrm{NO}_{3}\right)_{2} \cdot 3 \mathrm{H}_{2} \mathrm{O}$} & $\mathrm{C}_{44} \mathrm{H}_{58} \mathrm{~N}_{6} \mathrm{NiO}_{13}$ & \multirow{2}{*}{937} & \multirow{2}{*}{$>250$} & 56.36 & 6.23 & 8.96 & \\
\hline & 937.66 & & & 56.33 & 6.45 & 9.01 & \\
\hline \multirow{2}{*}{$\mathrm{Cd}(\mathrm{EN} 2)\left(\mathrm{NO}_{3}\right)_{2} \cdot 2 \mathrm{H}_{2} \mathrm{O}$} & $\mathrm{C}_{11} \mathrm{H}_{17} \mathrm{CdN}_{3} \mathrm{O}_{9}$ & \multirow{2}{*}{448} & \multirow{2}{*}{$>250$} & 29.51 & 3.83 & 9.39 & \\
\hline & 447.68 & & & 29.45 & 4.11 & 9.46 & \\
\hline \multirow{2}{*}{$\begin{array}{l}\mathrm{Hg}(\mathrm{EN} 2)_{2}\left(\mathrm{NO}_{3}\right)_{2} \cdot 10 \mathrm{H}_{2} \\
\mathrm{O}\end{array}$} & $\mathrm{C}_{22} \mathrm{H}_{46} \mathrm{HgN}_{4} \mathrm{O}_{18}$ & \multirow{2}{*}{855} & \multirow{2}{*}{$>250$} & 30.90 & 5.42 & 6.55 & \\
\hline & 855.21 & & & 31.08 & 5.22 & 6.34 & \\
\hline $\mathrm{Pb}(\mathrm{EN} 2)_{2}\left(\mathrm{NO}_{3}\right)_{2}$ & $\mathrm{C}_{22} \mathrm{H}_{26} \mathrm{~N}_{4} \mathrm{O}_{8} \mathrm{~Pb}$ & 682 & $>250$ & 38.76 & 3.84 & 8.22 & \\
\hline & 681.66 & & & 38.66 & 3.58 & 8.01 & \\
\hline
\end{tabular}

Table 3. ${ }^{1} \mathrm{H}$ and ${ }^{13} \mathrm{C}$ NMR signals (ppm) of EN1, EN2 and their complexes

\begin{tabular}{|c|c|c|}
\hline Compound & ${ }^{1} \mathrm{H}$ NMR & ${ }^{13} \mathrm{C} \mathrm{NMR}$ \\
\hline EN1 & $\begin{array}{l}\text { 7.77-7.70, 7.14-7.12 } \\
\text { (thiophene-H); 7.69, } 5.80 \\
(\mathrm{CH}=\mathrm{CH}) ; 3.15,3.08 \\
(2 \mathrm{Me})\end{array}$ & $\begin{array}{l}179.08(\mathrm{CO}) ; 153.56(\mathrm{CH}=) ; \\
91.42(=\mathrm{CH}) ; 148.12,130.91 \text {, } \\
\text { 128.57, } 127.95 \text { (thiophene- } \\
\text { carbons); 44.40, } 44.30\left(\mathrm{NMe}_{2}\right)\end{array}$ \\
\hline $\mathrm{CuEN} 1\left(\mathrm{NO}_{3}\right)_{2}$ & $\begin{array}{l}\text { 8.02-7.40 (thiophene-H); } \\
\text { 7.77, } 5.40(\mathrm{CH}=\mathrm{CH}) ; 3.30 \text {, } \\
3.23(2 \mathrm{Me})\end{array}$ & $\begin{array}{l}188.77(\mathrm{CO}) ; 153.00(\mathrm{CH}=) ; \\
78.04(=\mathrm{CH}) ; 143.09,130.94 \\
\text { 128.84, } 127.89 \text { (thiophene- } \\
\text { carbons);39.50, } 39.48\left(\mathrm{Nme}_{2}\right)\end{array}$ \\
\hline $\mathrm{Co}(\mathrm{EN} 1)_{4}\left(\mathrm{NO}_{3}\right)_{2}$ & $\begin{array}{l}\text { 7.93-7.73, 7.15-7.16 } \\
\text { (thiophene-H); 7.66, } 5.76 \\
(\mathrm{CH}=\mathrm{CH}) ; 3.10,2.87 \\
(2 \mathrm{Me})\end{array}$ & $\begin{array}{l}185.83(\mathrm{CO}) ; 148.56(\mathrm{CH}=) ; \\
85.79(=\mathrm{CH}) ; 143.52,130.14, \\
\text { 129.86, } 125.89 \text { (thiophene- } \\
\text { carbons); 39.56, } 39.36\left(\mathrm{Nme}_{2}\right)\end{array}$ \\
\hline $\begin{array}{l}\mathrm{Ni}(\mathrm{EN} 1)_{3} \\
2 \mathrm{H}_{2} \mathrm{O}\end{array}$ & $\begin{array}{l}\text { 7.52-7.34, 7.15-7.16 } \\
\text { (thiophene-H); 7.26, } 5.35 \\
(\mathrm{CH}=\mathrm{CH}) ; 3.19,2.72 \\
(2 \mathrm{Me})\end{array}$ & $\begin{array}{l}183.24(\mathrm{CO}) ; 145.88(\mathrm{CH}=) ; \\
83.04(=\mathrm{CH}) ; 140.78,136.59, \\
\text { 123.41, } 121.06 \text { (thiophene- } \\
\text { carbons); 39.59, } 39.39\left(\mathrm{Nme}_{2}\right)\end{array}$ \\
\hline $\mathrm{CdEN} 1\left(\mathrm{NO}_{3}\right)_{2}$ & $\begin{array}{l}\text { 8.42-7.91, 7.76-7.33 } \\
\text { (thiophene-H); 7.67, } 5.80 \\
(\mathrm{CH}=\mathrm{CH}) ; 3.13,2.90 \\
(2 \mathrm{Me})\end{array}$ & $\begin{array}{l}185.87(\mathrm{CO}) ; 142.07(\mathrm{CH}=) ; \\
\text { 85.14 }(=\mathrm{CH}) ; 138.16,136.85 \\
\text { 132.26, } 128.75 \text { (thiophene- } \\
\text { carbons); 40.12, } 39.92\left(\mathrm{Nme}_{2}\right)\end{array}$ \\
\hline
\end{tabular}




\begin{tabular}{|c|c|c|}
\hline $\operatorname{HgEN1}\left(\mathrm{NO}_{3}\right)_{2}$ & $\begin{array}{l}\text { 8.42-7.09 (thiophene-H); } \\
\text { 7.26, } 5.45(\mathrm{CH}=\mathrm{CH}) \\
\text { 3.13, } 2.89(2 \mathrm{Me})\end{array}$ & $\begin{array}{l}188.00(\mathrm{CO}) ; 142.07(\mathrm{CH}=) ; \\
85.14(=\mathrm{CH}) ; 138.16,129.40, \\
\text { 129.12, 128.75 (thiophene- } \\
\text { carbons); 4013, } 39.92\left(\mathrm{NMe}_{2}\right)\end{array}$ \\
\hline $\mathrm{PbEN} 1\left(\mathrm{NO}_{3}\right)_{2}$ & $\begin{array}{l}\text { 8.41-7.30 (thiophene-H); } \\
\text { 7.32, } 5.45(\mathrm{CH}=\mathrm{CH}) \\
\text { 3.40, } 3.41(2 \mathrm{Me})\end{array}$ & $\begin{array}{l}185.89(\mathrm{CO}) ; 142.09(\mathrm{CH}=) ; \\
85.23(=\mathrm{CH}) ; 138.17,136.79, \\
\text { 132.33, 129.15 (thiophene- } \\
\text { carbons); 40.12, } 39.91\left(\mathrm{NMe}_{2}\right)\end{array}$ \\
\hline EN2 & $\begin{array}{l}\text { 7.97-7.90, 7.58-7.40 (Ar- } \\
\mathrm{H}) ; 7.76,5.85(\mathrm{CH}=\mathrm{CH}) \\
\text { 2.89 (2Me) }\end{array}$ & $\begin{array}{l}185.71(\mathrm{CO}) ; 154.24(\mathrm{CH}=) ; \\
\text { 91.69 (=CH); 136.77, 130.76, } \\
\text { 128.55, 126.52 (Ar-carbons); } \\
\text { 44.47, 44.35 }\left(\mathrm{NMe}_{2}\right)\end{array}$ \\
\hline $\mathrm{CuEN} 2\left(\mathrm{NO}_{3}\right)_{2}$ & $\begin{array}{l}\text { 7.75-7.33(Ar-H); } 7.76, \\
5.85(\mathrm{CH}=\mathrm{CH}) ; 2.92 \\
(2 \mathrm{Me})\end{array}$ & $\begin{array}{l}178.52(\mathrm{CO}) ; 146.01(\mathrm{CH}=) ; \\
\text { 91.60 (=CH); } 143.19,131.71 \text {, } \\
\text { 124.67, 122.70 (Ar-carbons); } \\
\text { 39.48, 39.36(}\left(\mathrm{NMe}_{2}\right)\end{array}$ \\
\hline $\mathrm{Co}(\mathrm{EN} 2)_{4}\left(\mathrm{NO}_{3}\right)_{2}$ & $\begin{array}{l}\text { 8.02-7.92, 7.39-7.24 (Ar- } \\
\mathrm{H}) ; 7.69,5.86(\mathrm{CH}=\mathrm{CH}) \\
2.93(2 \mathrm{Me})\end{array}$ & $\begin{array}{l}170.95(\mathrm{CO})) ; 158.52(\mathrm{CH}=) ; \\
\text { 85.42 (=CH); 130.81, 125.23; } \\
\text { 123.07, 121.86 (Ar-carbons); } \\
\text { 39.67, 39.49(}\left(\mathrm{NMe}_{2}\right)\end{array}$ \\
\hline $\mathrm{Ni}(\mathrm{EN} 2)_{4}\left(\mathrm{NO}_{3}\right)_{2} \cdot 3 \mathrm{H}_{2} \mathrm{O}$ & $\begin{array}{l}10.34\left(\mathrm{br} \mathrm{H}_{2} \mathrm{O}\right) ; 8.51- \\
\text { 7.71, 7.51-7.25 (Ar-H); } \\
\text { 7.68, 5.62 (CH=CH); } 2.95 \\
(2 \mathrm{Me})\end{array}$ & $\begin{array}{l}170.95(\mathrm{CO})) ; 154.23(\mathrm{CH}=) ; \\
75.19(=\mathrm{CH}) ; 149.94,128.11 \text {, } \\
\text { 127.61, 124.47, 122.97 (Ar- } \\
\left.\text { carbons); 39.52, 39.53( } \mathrm{NMe}_{2}\right)\end{array}$ \\
\hline $\mathrm{Cd}(\mathrm{EN} 2)\left(\mathrm{NO}_{3}\right)_{2} \cdot 2 \mathrm{H}_{2} \mathrm{O}$ & $\begin{array}{l}10.34\left(\mathrm{br} \mathrm{H}_{2} \mathrm{O}\right) ; 8.32- \\
\text { 7.84, 7.45-7.25 }(\mathrm{Ar}-\mathrm{H}) \\
\text { 7.84, 5.62 }(\mathrm{CH}=\mathrm{CH}) \\
\text { 3.35, 3.32 }(2 \mathrm{Me})\end{array}$ & $\begin{array}{l}172.35(\mathrm{CO})) ; 153.23(\mathrm{CH}=) ; \\
\text { 79.20 (=CH); 137.77, 131.76, } \\
\text { 129.34, 126.52 (Ar-carbons); } \\
\text { 44.45, 44.40 }\left(\mathrm{NMe}_{2}\right)\end{array}$ \\
\hline $\mathrm{Hg}(\mathrm{EN} 2)_{2}\left(\mathrm{NO}_{3}\right)_{2} \cdot 10 \mathrm{H}_{2} \mathrm{O}$ & $\begin{array}{l}9.87\left(\text { br } \mathrm{H}_{2} \mathrm{O}\right) ; 8.27-7.86, \\
7.55-7.41(\mathrm{Ar}-\mathrm{H}) ; 7.63, \\
5.82(\mathrm{CH}=\mathrm{CH}) ; 3.40,3.16 \\
(2 \mathrm{Me})\end{array}$ & $\begin{array}{l}177.35(\mathrm{CO})) ; 154.23(\mathrm{CH}=) ; \\
\text { 80.20 (=CH); 137.77, 131.76, } \\
\text { 128.49, 126.52 (Ar-carbons); } \\
\text { 40.12, 39.91 }\left(\mathrm{NMe}_{2}\right)\end{array}$ \\
\hline $\mathrm{Pb}(\mathrm{EN} 2)_{2}\left(\mathrm{NO}_{3}\right)_{2}$ & $\begin{array}{l}\text { 8.25-7.70, 7.64-7.28 (Ar- } \\
\mathrm{H}) ; 7.68,5.82(\mathrm{CH}=\mathrm{CH}) \\
3.47,3.18(2 \mathrm{Me})\end{array}$ & $\begin{array}{l}\text { 199.56, } 194.52(\mathrm{CO})) ; 154.23 \\
(\mathrm{CH}=) ; 80.20(=\mathrm{CH}) ; 137.57, \\
\text { 136.32, 134.02, 128.28 (Ar- } \\
\text { carbons); 40.13, } 39.92\left(\mathrm{NMe}_{2}\right)\end{array}$ \\
\hline
\end{tabular}

\section{Antimicrobial studies}

The in vitro antibacterial activity of enaminones and their complexes were evaluated against gram positive and gram negative bacteria, (Bacillus sp. M3010, Bacillus sp. M3017, Escherichia Coli-PA149, Escherichia Coli-PA151, Staphylococcus aureus-PA128, Staphylococcus aureus-PA129, Salmonella sp.-PA392 and Salmonella sp.-PA393) and three fungi (Candida albicans-PA47, Candida albicans-PA48 and Candida albicans-PA49). The hole plate diffusion method ${ }^{12}$ was adopted for the activity measurements. The bacterial strains were grown in nutrient agar slants. A suspension of the studied compounds $(0.2 \mathrm{~m} \mathrm{~L}$ of each $(10 \mu \mathrm{g} / \mathrm{mL})$ was incubated at $36{ }^{\circ} \mathrm{C}$ for $36 \mathrm{~h}$ for the bacterial culture. After inoculation, the diameter (in $\mathrm{mm}$ ) of the clear inhibition zone surrounding the sample is taken as a measure of the inhibition power against the particular organisms. The values recorded are the mean average for experiments repeated three times. 


\section{Results and Discussion}

\section{Characterization of Ligand}

${ }^{1} \mathrm{H}$ NMR of 3-(dimethylamino)-1-(thiophen-2-yl)prop-2-en-1-one (EN1) (cf. Scheme 1) reveals the presence of thiophene protons as doublet of doublet at $\delta$ 7.77-7.70 and 7.14 -7.12 ppm. The ethylenic proton appears at $\delta 7.69$ and $5.80 \mathrm{ppm}$ in addition, the $\mathrm{NMe}_{2}$ protons appeared at $\delta 3.08$ and 3.15 ppm. ${ }^{13} \mathrm{C}$ NMR showed peaks at $\delta 179.08$ (CO); 153.56 (CH=); 91.42 (=CH); 148.12, 130.91, 128.57, 127.95 (thiophene-carbons); 44.40, $44.30\left(\mathrm{NMe}_{2}\right)$. IR showed the carbonyl group at $1653 \mathrm{~cm}^{-1}$ other characteristic bands reported in Table 1 . The mass spectrum showed the molecular ion peak at $\mathrm{m} / \mathrm{z} 181\left(\mathrm{M}^{+}\right)$with fragments at $\mathrm{m} / \mathrm{z} 111$ for thionylium ion $\mathrm{C}_{5} \mathrm{H}_{3} \mathrm{OS}^{*}$ with other fragments at $\mathrm{m} / \mathrm{z}$ 164, 148 and 70.

The mass spectrum of 3-(dimethylamino)-1-phenylprop-2-en-1-one (EN2) (cf. Scheme 1) showed the molecular ion peak at $m / z 175$ with different fragments at 158, 131, 98 and 77. ${ }^{1} \mathrm{H}$ NMR reveals the presence of aromatic protons at $\delta 7.97-7.90$ and 7.58-7.41 ppm.

The ethylenic proton appears at $\delta 7.76$ and $5.85 \mathrm{ppm}$ in addition, the $\mathrm{NMe}_{2}$ protons appeared at $\delta 2.89 \mathrm{ppm} .{ }^{13} \mathrm{C}$ NMR showed peaks at $\delta 185.71$ for carbonyl group; 154.24 and 91.69 for ethylenic carbons; 136.77, 130.76, 128.55, 126.52 for aromatic carbons; 44.47, 44.35 for two methyl groups of $\mathrm{NMe}_{2}$. IR showed the carbonyl group at $1650 \mathrm{~cm}^{-1}$, in addition to other characteristic bands ( $c f$. Table 1)

\section{Characterization of the complexes}

Both EN1 and EN2 could be chelate from two centers, carbonyl group and $\mathrm{NH}$ group as a bidentate system. Different complexes were formed on the reaction of EN1 or EN2 with the nitrate salts of $\mathrm{Cu}(\mathrm{II}), \mathrm{Co}(\mathrm{II}), \mathrm{Ni}(\mathrm{II}), \mathrm{Pb}(\mathrm{II}), \mathrm{Hg}(\mathrm{II})$ and $\mathrm{Cd}(\mathrm{II})$. The IR spectra of the complexes showed that EN1 and EN2 are coordinate in the neutral bidentate mode. Bands at 1650 to $1659 \mathrm{~cm}^{-1}$ is assigned to carbonyl group and that at 1545 to $1596 \mathrm{~cm}^{-1}$ is assigned to C-N. Moreover, aromatic protons appeared in peaks more than $3000 \mathrm{~cm}^{-1}$ ( $c f$. Table 1). The elemental analysis and spectral data results ( $c f$. Tables 1-3) showed the formation of CuEN1 $\left(\mathrm{NO}_{3}\right)_{2} ; \mathrm{CdEN} 1\left(\mathrm{NO}_{3}\right)_{2}, \mathrm{HgEN} 1\left(\mathrm{NO}_{3}\right)_{2}, \mathrm{PbEN} 1\left(\mathrm{NO}_{3}\right)_{2}$, Scheme 1 structure A, while $\mathrm{Ni}(\mathrm{II})$ have the structure $\mathrm{Ni}(\mathrm{EN} 1)_{3}\left(\mathrm{NO}_{3}\right)_{2} \cdot \mathrm{H}_{2} \mathrm{O}$ Scheme 1 structure $\mathrm{C}$. EN2 complexes are $\mathrm{CuEN2}\left(\mathrm{NO}_{3}\right)_{2}$ (Scheme 1 structure A); $\mathrm{Co}(\mathrm{EN2})_{4}\left(\mathrm{NO}_{3}\right)_{2}$ and $\mathrm{Ni}(\mathrm{EN} 2)_{4}\left(\mathrm{NO}_{3}\right)_{2} \cdot 3 \mathrm{H}_{2} \mathrm{O}$ (Scheme 1 structure $\mathrm{D}) ; \mathrm{Cd}(\mathrm{EN} 2)\left(\mathrm{NO}_{3}\right)_{2} \cdot 25 \mathrm{H}_{2} \mathrm{O}$ (Scheme 1 structure A); $\mathrm{Hg}(\mathrm{EN} 2)_{2}\left(\mathrm{NO}_{3}\right)_{2} \cdot 10 \mathrm{H}_{2} \mathrm{O}$ and $\mathrm{Pb}(\mathrm{EN} 1)_{2}\left(\mathrm{NO}_{3}\right)_{2}$ (Scheme 1 structure B). All complexes obtained are colored either due d-d or charge transfer or charge transfer transitions.

\section{Biological activity}

The well-known diverse biological activities of enaminones ${ }^{13-18}$ prompted us to test and study the antibacterial and antifungal activities of the synthesized products. Table 4 shows that most of the tested compounds had moderate to high activity against most investigated microorganisms. All prepared compounds are investigated against gram positive and gram negative bacterial (microorganisms undergoes investigation are Bacillus sp. M3010; Bacillus sp. M3017; Escherichia Coli-PA149; Escherichia Coli-PA151; Staphylococcus aureusPA128; Staphylococcus aureus-PA129; Salmonella sp.-PA392 and Salmonella sp.-PA393) and antifungal (Candida albicans-PA47; Candida albicans- PA48 and Candida albicansPA49). The complexes Co-EN1-complex; Ni-EN1-complex; Co-EN2-complex and Pb-EN2complex have no any reactivity against all tested microorganisms (gram positive, gram negative and fungus). 

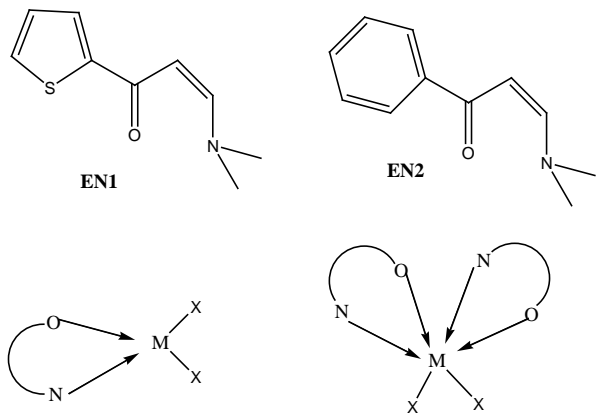

A

B

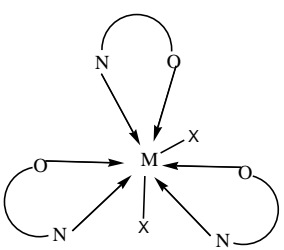

C

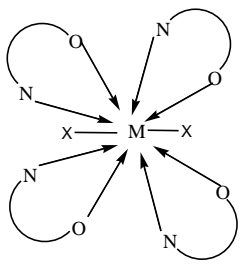

D

\section{Scheme 1}

Table 4. Antibacterial and antifungal activity of ligand and complexes

\begin{tabular}{|c|c|c|c|c|c|c|c|c|c|c|c|c|c|c|}
\hline \multicolumn{15}{|c|}{ Chemical compound } \\
\hline & & & & Zone & of in & hibiti & ion, $\mathrm{m}$ & & & & & & & \\
\hline Bacteria & EN1 & Co1 & Hg1 & Pb1 & Ni1 & Cu1 & Cd1 & EN2 & Co2 & Hg2 & Pb2 & Ni2 & Cu2 & Cd2 \\
\hline $\begin{array}{l}\text { Bacillus } \\
\text { sp. M3010 }\end{array}$ & 15 & Nil & 18 & 13 & Nil & Nil & 13 & 16 & Nil & 16 & Nil & Nil & 19 & Nil \\
\hline $\begin{array}{l}\text { Bacillus } \\
\text { sp. M3017 }\end{array}$ & 14 & Nil & 17 & 15 & Nil & Nil & 15 & 18 & Nil & 15 & Nil & Nil & 17 & Nil \\
\hline $\begin{array}{l}\text { Escherichia } \\
\text { Coli-PA149 }\end{array}$ & 18 & Nil & 16 & 16 & Nil & Nil & 18 & 21 & Nil & 21 & Nil & Nil & Nil & Nil \\
\hline $\begin{array}{l}\text { Escherichia } \\
\text { Coli-PA151 }\end{array}$ & 16 & Nil & 15 & 15 & Nil & Nil & 17 & 19 & Nil & 19 & Nil & Nil & Nil & Nil \\
\hline $\begin{array}{l}\text { Staphylococcus } \\
\text { aureus-PA128 }\end{array}$ & Nil & Nil & 21 & 21 & Nil & Nil & 16 & Nil & Nil & 18 & Nil & Nil & 19 & Nil \\
\hline $\begin{array}{l}\text { Staphylococcus } \\
\text { aureus-PA129 }\end{array}$ & Nil & Nil & 20 & 20 & Nil & Nil & 18 & Nil & Nil & 18 & Nil & Nil & 18 & Nil \\
\hline $\begin{array}{l}\text { Salmonella sp.- } \\
\text { PA392 }\end{array}$ & 16 & Nil & 16 & 16 & Nil & Nil & 21 & 17 & Nil & 19 & Nil & Nil & 16 & Nil \\
\hline $\begin{array}{l}\text { Salmonella sp.- } \\
\text { PA393 }\end{array}$ & 17 & Nil & 18 & 18 & Nil & Nil & 20 & 16 & Nil & 18 & Nil & Nil & 15 & Nil \\
\hline $\begin{array}{l}\text { Candida } \\
\text { albicans-PA47 }\end{array}$ & 20 & Nil & 19 & 23 & Nil & 16 & 16 & 18 & Nil & 21 & Nil & Nil & 21 & 23 \\
\hline $\begin{array}{l}\text { Candida } \\
\text { albicans- PA48 }\end{array}$ & 18 & Nil & 17 & 21 & Nil & 15 & 18 & 19 & Nil & 23 & Nil & $\mathrm{Nil}$ & 20 & 21 \\
\hline $\begin{array}{l}\text { Candida } \\
\text { albicans- PA49 }\end{array}$ & 17 & Nil & 15 & 22 & Nil & 18 & 19 & 18 & Nil & 21 & Nil & Nil & 18 & 20 \\
\hline
\end{tabular}


The data of EN1 and its complexes with different metal nitrate salts against gram positive bacteria can be summarized as follows: Bacillus sp. M3010 shows that the Hg has highest effect according to the following order $\mathrm{Hg}^{2+}>\mathrm{EN} 1>\mathrm{Pb}^{2+}=\mathrm{Cd}^{2+}$. Similarly, Bacillus $s p$. M3017 shows highest effect with $\mathrm{Hg}$ according to the order $\mathrm{Hg}>\mathrm{Pb}=\mathrm{Cd}>\mathrm{EN1}$.

Furthermore, both types of Staphylococcus aureus-PA128 and Staphylococcus aureusPA129 showed high effect in case of $\mathrm{Hg}$ and $\mathrm{Pb}$ and both of them are equal in its effect. The order according to the inhibition zone can arranged as follows, Staphylococcus aureusPA128: $\mathrm{Pb}=\mathrm{Hg}>\mathrm{Cd}$ and Staphylococcus aureus-PA129: $\mathrm{Pb}=\mathrm{Hg}>\mathrm{Cd}$.

The effect of tested compounds against gram negative bacteria can be summarized as follows: Escherichia Coli-PA149 shows the highest effect with the ligand and can be order as follows, EN1 $>\mathrm{Cd}^{2+}>\mathrm{Hg}^{2+}=\mathrm{Pb}^{2+}$, while the highest effect of Escherichia Coli-PA151 observed with Cd complex and ordered as follows $\mathrm{Cd}>\mathrm{EN} 1>\mathrm{Pb}=\mathrm{Hg}$.

Both Salmonella sp.-PA392 and Salmonella sp.-PA393 are showed approximately the same effect with the tested compounds and can ordered with Salmonella sp.-PA392 as follows: $\mathrm{Cd}>\mathrm{Pb}=\mathrm{Hg}=\mathrm{EN1}$ and with Salmonella sp.-PA393 as follows: $\mathrm{Cd}>\mathrm{Pb}=\mathrm{Hg}>\mathrm{EN1}$.

All tested fungi highly effected by $\mathrm{Pb}$ complex and can be arranged as follows: in case of Candida albicans-PA47: $\mathrm{Pb}>\mathrm{EN} 1>\mathrm{Hg}>\mathrm{Cu}=\mathrm{Cd}$; in case of Candida albicans-PA48: $\mathrm{Pb}>$ $\mathrm{Cd}>\mathrm{Cu}>\mathrm{EN} 1>\mathrm{Hg}$ and in case of Candida albicans-PA49: $\mathrm{Pb}>\mathrm{Cd}>\mathrm{Cu}>\mathrm{EN} 1>\mathrm{Hg}$

The data of EN1 and its complexes with different metal nitrate salts against gram negative bacteria can be ordered as follows: Bacillus sp. M3010: $\mathrm{Cu}>\mathrm{Hg}=\mathrm{EN} 2$; while in case of Bacillus sp. M3017: EN2 > Cu > Hg. In case of Staphylococcus aureusPA128: $\mathrm{Cu}>\mathrm{Hg}$ and with Staphylococcus aureus-PA129 showed similar effect with $\mathrm{Cu}$ $=\mathrm{Hg}$. On the other hand, Escherichia Coli-PA149 and Escherichia Coli-PA151 are showed the same effect with EN2 and Hg-complex, EN2= Hg. Both types tested from Salmonella sp.-PA392 and Salmonella sp.-PA393 are showed the same effect on tested compounds, order as follows $\mathrm{Hg}>\mathrm{EN} 2>\mathrm{Cu}$. All tested fungi against EN2 and its complexes are showed highly effected by $\mathrm{Hg}$ and $\mathrm{Cd}$ complexes and can be arranged as follows: in case of Candida albicans-PA47: $\mathrm{Cd}>\mathrm{Cu}=\mathrm{Hg}>\mathrm{EN2}$; in case of Candida albicans-PA48: $\mathrm{Hg}>\mathrm{Cd}>\mathrm{Cu}>\mathrm{EN} 2$ and in case of Candida albicans-PA49: $\mathrm{Hg}>\mathrm{Cd}$ $>\mathrm{Cu}=\mathrm{EN} 2$

Generally the highest effected was observed in case of EN1 complexes as compared by that of EN2. This may be due the presence of thiophene ring in EN1- complexes as compared by phenyl group in EN2-complexes. High effect against different types of tested fungi was observed in case of Cd-EN2-complex, Pb-EN1-complex, Cd-EN2-complex, HgEN2-complex and Cu-EN2-complex. High effect against Escherichia Coli-PA149 was observed in case of Hg-EN2-complex, while Hg-EN1-complex showed high effect against Staphylococcus aureus-PA128 and Staphylococcus aureus-PA129. Salmonella sp.-PA392 and Salmonella sp.-PA393 showed high effect with Cd-EN1-complex.

\section{Conclusion}

In the light of the data one can conclude the following:

- Enaminone acts as a good chelating agent in aqueous ethanolic solution.

- Bioactivity of enaminones and most of their complexes showed a moderate to high effect on the tested bacteria and fungi.

- The enaminone which containing thiophene ring more effective on microorganisms rather than that containing aromatic phenyl group. 


\section{Acknowledgement}

The authors are grateful to the Chemistry Department, and the Faculty of Science Kuwait University, and for the SAF facilities

\section{References}

1. Elassar A Z and El-Khair A, Tetrahedron, 2003, 59(43), 8463-8480; DOI:10.1016/S0040-4020(03)01201-8

2. $\quad$ Liu Y, Wang C, Wang X and Wan J P, Tetrahedron Lett., 2013, 54(30), 3953-3955; DOI:10.1016/j.tetlet.2013.05.063

3. Samantaray M K, Dash C, Shaikh M M, Pang K, Butcher R J and Ghosh P, Inorg Chem., 2011, 50(5), 1840-1848; DOI:10.1021/ic102268n

4. Vicente J, Chicote M T, Martı'nez-Martı'nez A J and Abell_an-L_opez A, Organometallics, 2010, 29(21), 5693-5707; DOI:10.1021/om1005403

5. Shi Y C and Hu Y Y, J Coord Chem., 2009, 62(8), 1302-1312; DOI:10.1080/00958970802573028

6. Shi Y C, Cheng H J and Zhang S H, Polyhedron, 2008, 27(16), 3331-3336; DOI:10.1016/j.poly.2008.04.057

7. Shi Y C, Yang H M, Song H B and Liu Y H, Polyhedron, 2004, 23(9), 1541-1546; DOI:10.1016/j.poly.2004.04.002

8. $\quad$ Singh S J and Singh O M, Tetrahedron Lett., 2008, 49(25) , 3991-3994; DOI:10.1016/j.tetlet.2008.04.093

9. Concetta M, J Brazilian Chem Soc., 2003, 14(6), 945-969; DOI:10.1590/S010350532003000600012

10. Mahmud T, Rehman R, Gulzar A, Khalid A, Anwar J, Shafique U, Waheed-uzZaman and Salman M, Arab J Chem., 2010, 3(4), 219-224; DOI:10.1016/j.arabjc.2010.06.003

11. Zhang T, Jia Y M, Yan S J, Yu C Y and Huang Z T, ARKIVOC, 2009, xiv, 156-170.

12. Collins C H, Lyne P M, Gillard In McCleverty R D J A (Ed.), 1970 "Microbiol-ogical Methods "Univeristy Park Press, Baltimore, MD.

13. Liu Y X, Zhao H P, Wang Z W, Li Y H, Song H B, Riches H, Beattie D, Gu Y C and Wang Q M, Molecular Diversity, 2013, 17(4), 701-710; DOI:10.1007/s11030-0139466-6

14. Kathrotiya H G and Patel P M, Eur J Med Chem., 2013, 63, 675-684;

DOI:10.1016/j.ejmech.2013.03.017

15. Martinez J C G, Duchowicz P R, Estrada M R, Zamarbide G N and Castro E, QSAR Comb Sci., 2009, 28(11-12), 1376-1385.

16. Edafiogho I O, Kombia S B, Ananthalakshmi K V V, Salama N N, Eddington N D, Wilson T L, Alexander M S, Jackson P L. Hanson C D and Scott K R, J Pharm Sci., 2007, 96(10), 2509-2531; DOI:10.1002/jps.20967

17. Al-Omran F, Elassar A Z and El-Khair A A, Tetrahedron, 2001, 57(51), 1016310170; DOI:10.1016/S0040-4020(01)01039-0

18. Scott K R, Nicholson J M and Edafiogho I O, U.S. (1995), US 5468775 A19951121. 